\title{
Bias and Negligence with Freedom of Information*
}

\author{
Rossella Argenziano ${ }^{\dagger}$ and Helen Weeds ${ }^{\ddagger}$
}

September 2018

\begin{abstract}
We analyse decision-making in the presence of Freedom of Information (FOI) rules. A decision-maker chooses whether to acquire costly information to inform his decision regarding a policy action. If information is not disclosed voluntarily a monitor may open a costly investigation, using FOI to access the information. A finding of biased decision-making or negligence in information acquisition generates a reward to the monitor and a penalty to the decision-maker. We find that strengthening FOI to reduce the cost of investigation may increase negligence without necessarily reducing bias. Moreover increasing the reward for discovering negligence can paradoxically increase negligence in equilibrium.
\end{abstract}

\footnotetext{
${ }^{*}$ We would like to thank Bernard Caillaud, Claude d'Aspremont, Stephen Hansen, Bruno Jullien, François Maniquet, Santiago Oliveros, Chris Wallace, Piercarlo Zanchettin, and conference and seminar participants at TSE Eighth bi-annual conference on The Economics of Intellectual Property, Software and the Internet, UECE Lisbon Game Theory Meetings, CORE Université Catholique de Louvain, University of Leicester and University of Essex.

${ }^{\dagger}$ University of Essex. Email: rargenz@essex.ac.uk.

${ }^{\ddagger}$ Imperial College Business School. Email: helen.weeds@gmail.com.
} 
Freedom of Information (FOI) legislation establishes the principle that citizens, including journalists and interested parties, should be able to access any document held by a public body. Its goal is to discipline officials to act in the public interest rather than following their private desires or pandering to favoured groups. The first FOI law was the Swedish Freedom of the Press Act of 1766. Two hundred years later the United States passed its Freedom of Information Act (FOIA). Currently, over one hundred countries around the world have adopted FOI regimes (McIntosh, 2014).

FOI legislation continues to evolve and its principles occasionally come under threat. The U.S. FOIA Improvement Act of 2016 limits the exemptions that government agencies can invoke to deny access to documents and establishes a 'presumption of openness' (The Washington Post, 2016). In the U.K., the Independent Commission on Freedom of Information, set up in July 2015 to examine concerns that the Freedom of Information Act 2000 inhibits the workings of government, reported in March 2016 that 'there is no evidence that the Act needs to be radically altered, or that the right of access to information needs to be restricted' (see Cabinet Office, 2016; also Allen and Pickard, 2016 and Quinn, 2016). Given the recent interest in FOI, the impact of such measures is a timely and important topic for consideration.

Our paper highlights the impact of FOI (and other, e.g. court-based, disclosure processes) on the incentive for public decision-makers to acquire relevant information prior to taking decisions as required to weigh up the options as accurately as possible. When information that is gathered might later be subject to a FOI request, with adverse revelations having detrimental consequences for the decision-maker, there may be a 'chilling' effect on information acquisition. The paper also investigates institutional design of FOI to achieve desirable discipline effect on decision-making while minimising detrimental impacts on information acquisition. 
We present a model in which a decision-maker makes a binary choice of action $a \in\{0,1\}$. The resulting social benefit depends on the state of the world, which is unknown at the start of the game, while the decisionmaker also receives a private benefit from $a=1$ which biases his choice. Prior to choosing, the decision-maker may acquire information revealing the state of the world; this may be interpreted broadly to include not just data collection but also analysis, which similarly requires time and effort.

As a benchmark, we start by considering the privately optimal behaviour of a decision-maker in the absence of FOI, on the assumption that scrutiny is prohibitively expensive in its absence. The private benefit from $a=1$ reduces social welfare in two distinct ways. First, when information is acquired the probability of taking the socially inferior action (an occurrence we refer to as bias) is increasing in the private benefit. Secondly, the probability of not acquiring information (an outcome we term negligence) is increasing in the private benefit: by reducing the impact of the state of the world on the decision-maker's choice, a larger private benefit lowers the private value of information.

In the main model we introduce a monitor who may use FOI to access the decision-maker's information. Now, an informed decision-maker may choose to disclose his information at the same time as choosing the action. The monitor observes the action but, unless this is voluntarily revealed, she does not observe the information (if acquired), nor the fact of its acquisition. In the absence of voluntary disclosure the monitor may, at some cost, open an investigation in which she uses FOI to access the information held by the decision-maker. ${ }^{1}$ We assume that information is 'hard' in the

\footnotetext{
${ }^{1}$ The scope of FOI regimes is typically very wide, providing access to recorded information in all forms and covering both raw data and analysis of the data.
} 
sense that it cannot be hidden or distorted. ${ }^{2}$ If voluntary disclosure or an investigation show the decision-maker to be biased he incurs a punishment; a (weakly smaller) punishment is incurred if investigation reveals that he was negligent. Following an investigation the monitor receives a reward if she discovers bias and a (weakly smaller) reward if she uncovers negligence.

We find that the introduction of FOI has several effects on the inefficiencies identified in the benchmark. As one might expect, the threat of investigation and punishment has a positive discipline effect which consists of a reduction in bias, inducing the decision-maker to take the socially optimal action more frequently. The impact of FOI on information acquisition is more complex, however: if the punishment for negligence is small, FOI has a chilling effect which consistes of an aggravation of negligence. By disciplining his action choice and punishing bias, the threat of investigation reduces the decision-maker's payoff from being informed, thus decreasing his incentive to acquire information in the first place. Strengthening FOI to reduce the cost of an investigation, if carried out in isolation, may increase negligence without necessarily reducing bias; paradoxically, bias may even be increased.

We identify the first best institutional design, assuming that the legislator can control not only the investigation cost but also all of the punishment and reward parameters. We then explore the second best outcomes that may be achieved when a legislator can affect only a few of the parameters. We find that the effectiveness of FOI depends critically on the magnitudes of the punishment for negligence and the reward for uncovering this, relative to those for bias. Increasing this punishment towards its first best

\footnotetext{
${ }^{2}$ Failing to record information or retaining it in a form that side-steps FOI requests may be forbidden by law: for example the U.S. Government in the Sunshine Act of 1976 requires government agencies to keep transcripts of 'meetings', defined to include discussions that effectively predetermine official actions. Secondly, attempts to circumvent FOI are likely to degrade the quality of information and undermine its use in decisionmaking: see Pickard and Stacey (2015) regarding the automatic deletion of emails sent from the U.K. Prime Minister's office, apparently in response to the introduction of FOI.
} 
level is always beneficial in reducing negligence. By contrast, when the punishment for negligence is small raising the reward for discovering it can aggravate negligence with no offsetting reduction in bias. A modest increase in the punishment for bias may also worsen negligence.

The paper provides insights into many real-world situations. We describe three specific applications - the media and political accountability, discovery in court proceedings and product safety approval-interpreting the players and parameters in each case and drawing out implications for policy.

The paper is structured as follows. Section 1 discusses related literature. Section 2 describes the model and benchmark results. Equilibrium analysis is given in Section 3. Section 4 presents the first best solution and second best institutional design. Section 5 discusses the three applications. Section 6 concludes. An appendix presents the full characterisation of equilibrium and its proof.

\section{Related literature}

The paper is related to several strands of literature. One is the literature on costs and benefits of transparency in the political process based on principal-agent theory. In a situation of moral hazard with complete contracting, Holmström (1979) demonstrates that the principal is never harmed by (and generally gains from) observing additional information about the agent's performance. In the absence of complete contracts, however, inefficiencies can arise, as shown by e.g. Maskin and Tirole (2004).

A number of papers consider the impact of transparency using models of career concerns. In Prat (2005) an agent may ignore his private signal, even if this means taking the inferior action, because the principal makes an inference about his ability from the action choice itself. Because of 
this inefficiency the principal's expected payoff may be higher when the action is concealed and only its consequence is observed. Based on this result Prat makes the policy recommendation that actions should not be revealed, a principle seen in several FOI regimes which allow short-term secrecy while the decision process is ongoing. The inefficiency in Prat's paper can be compared to those in ours as follows: in his model the signal is obtained automatically but may be ignored in equilibrium, whereas in ours information may not be acquired at all (negligence) or, when acquired, may be ignored for some realisations (bias). Hoppe (2013) sets out an adverse selection model where the agent can gather private information before the principal offers the contract and finds that the principal may be better off when information gathering is hidden. Levy (2007) considers decision-making in committees, analyzing the impact of transparency when committee members are motivated by career concerns.

Since the decision-maker in our model has the option of voluntarily disclosing his information, our analysis is also related to the literature on persuasion. On this literature see Milgrom (1981), Milgrom and Roberts (1986) and, more recently, Mathis (2008), Che and Kartik (2009), Rayo and Segal (2010), Kamenica and Gentzkow (2011, 2016), Felgenhauer and Loerke (2017), Felgenhauer and Schulte (2014) and Hagenbach, Koessler and Perez-Richet (2014); see also Milgrom (2008) for a survey. In common with papers on persuasion, and in contrast with 'cheap talk', in our analysis information is taken to be 'hard' and cannot be distorted. However, our paper differs from the persuasion literature in two respects. First, information has a private value to the sender (here, the decision-maker), who himself takes an action, distinct from its value in communication with the receiver (here, the monitor). Secondly, the receiver faces a cost of 'punishing' a sender who does not disclose information (investigation is costly). As a result, the unravelling argument that typically guarantees full disclosure 
of information in persuasion games does not apply to our model.

The characteristics of FOI legislation internationally are described by Frankel (2001). FOI's effects on U.K. government are discussed by Hazell and Glover (2011), Hazell, Bourke and Worthy (2012) and Worthy and Hazell (2013). The impact of transparency on the decision-making process is studied in a number of empirical papers, drawing on the natural experiment provided by the release of transcripts of the Federal Open Market Committee in 1993: see Meade and Stasavage (2008) and Hansen, McMahon and Prat (2017). Both papers find that transparency reduces dissent from the Chairman's policy proposal, increasing the conformity of opinions, while Hansen et al. also find evidence of greater information acquisition between meetings by inexperienced members of the committee.

\section{The model}

\subsection{Information acquisition without monitoring}

As a benchmark, we start by considering information acquisition by a decision-maker in the absence of monitoring. A decision-maker, D, chooses an action $a \in\{0,1\}$. The social payoff from his choice depends on the state of the world $\theta$, whose realisation is initially unknown to all parties. The distribution of $\theta$ is commonly known to be $\theta \sim U[0,1]$. Social welfare from action $a$ is given by $W(a, \theta)=-|a-\theta|$. The socially optimal decision rule $\alpha^{*}(\theta)$ is therefore

$$
\alpha^{*}(\theta)=\left\{\begin{array}{cc}
1 & \text { if } \theta>\frac{1}{2} \\
0 & \text { if } \theta<\frac{1}{2} \\
\{0,1\} & \text { if } \theta=\frac{1}{2}
\end{array}\right.
$$


If the realisation of $\theta$ is unknown the optimal action is assessed according to the prior distribution; society is then indifferent between $a=1$ and $a=0$.

Although D takes social welfare into account, he also receives a private benefit $b$ from $a=1$. D's payoff is given by $U(a, \theta, b)=a b-|a-\theta|$, where $b \in(0,1]$. Before choosing $a, \mathrm{D}$ can learn the realisation of $\theta$ at a cost $k>0 .{ }^{3}$ We will refer to $\theta$ as the 'type' of an informed decision-maker. To summarise, the timeline is as follows:

1. Nature chooses the state of the world $\theta$; this is unobservable.

2. D chooses whether or not to pay $k$ to learn the realisation of $\theta$.

3. D chooses action $a \in\{0,1\}$; payoffs are realised.

If informed, $\mathrm{D}$ makes his decision conditionally on $\theta$, using decision rule $\alpha(\theta)$. D optimally chooses action 1 if and only if $\theta$ weakly exceeds the privately optimal type threshold $\theta_{0} \equiv \frac{1-b}{2}$; in case of indifference, the decision-maker chooses $a=1$. As illustrated in Figure 1, $\theta_{0}$ is less than one-half, the socially optimal action threshold. Due to the private benefit $b>0$ there is an interval $\left[\theta_{0}, \frac{1}{2}\right)$ over which he chooses $a=1$ despite this being the socially inferior choice. The larger is $b$, the greater this interval. Notice that the private benefit operates in one direction only, thus there is no corresponding interval in which $\mathrm{D}$ chooses $a=0$ when $a=1$ is socially optimal: $\alpha(\theta)=\alpha^{*}(\theta)$ for $\theta \geq \frac{1}{2}$. The inefficiency whereby $\mathrm{D}$ is informed but fails to implement the socially optimal decision rule is termed bias.

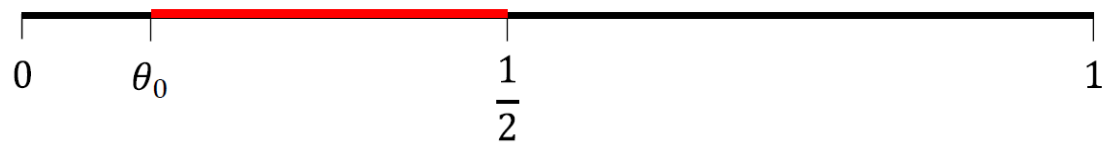

Fig. 1: Privately optimal decision rule

\footnotetext{
${ }^{3}$ We assume that information acquisition perfectly reveals the state of the world and study the impact of FOI on the probability that information is acquired. Alternatively, one could assume that information consists of an imperfect signal of the state and that the accuracy of the signal increases with expenditure, and study the impact of FOI on the precision of the information acquired.
} 
If uninformed, D maximises his expected utility given the prior. Given $b$, he therefore chooses $a=1$. Notice that since $\theta_{0}>0$, the interval over which the socially inferior action is chosen is greater when the decisionmaker is not informed than when he is. Put another way, the uninformed decision-maker's action choice is equivalent to the limiting case of bias where $b \rightarrow 1$.

Given the privately optimal action choice, the value of information to D, i.e. the difference between his expected utility when he acquires information and when he does not, is given by

$$
\int_{0}^{\theta_{0}}-\theta d \theta+\int_{\theta_{0}}^{1}[b-(1-\theta)] d \theta-\int_{0}^{1}[b-(1-\theta)] d \theta=\frac{(1-b)^{2}}{4} \equiv k_{0} .
$$

$k_{0}$ represents the value of hidden information: this is the maximum $\mathrm{D}$ is willing to pay to acquire information when both the acquisition of information and its content cannot be investigated by a third party. In the absence of monitoring information acquisition is optimal for D if and only if $k \leq k_{0}$. When acquired, information is used following the privately optimal action threshold $\theta_{0}$. Notice that greater $b$ reduces the value of hidden information, as information about $\theta$ makes less difference to D's action choice. In the limit where $b=1, \mathrm{D}$ acts regardless of the realisation of $\theta$ and $k_{0}=0$.

Next we determine the socially optimal information acquisition rule. The social value of information compares expected welfare when D acquires information and follows the socially optimal decision rule with expected welfare when D is uninformed:

$$
\left(\int_{0}^{\frac{1}{2}}-\theta d \theta+\int_{\frac{1}{2}}^{1}-(1-\theta) d \theta\right)-\int_{0}^{1}-(1-\theta) d \theta=\frac{1}{4} \equiv k^{* *}
$$

In the first best, information is acquired iff its cost does not exceed $k^{* *}$. For strictly positive $b, k^{* *}>k_{0}$ : the social value of information exceeds its 
private value and $\mathrm{D}$ underinvests in information, an inefficiency we term negligence. This is set out in Proposition 1 and illustrated in Figure 2.

Proposition 1 (Information acquisition without monitoring)

In the absence of monitoring, greater private benefit reduces the decisionmaker's incentive to acquire information. Compared with the social optimum, the decision-maker underinvests in information.

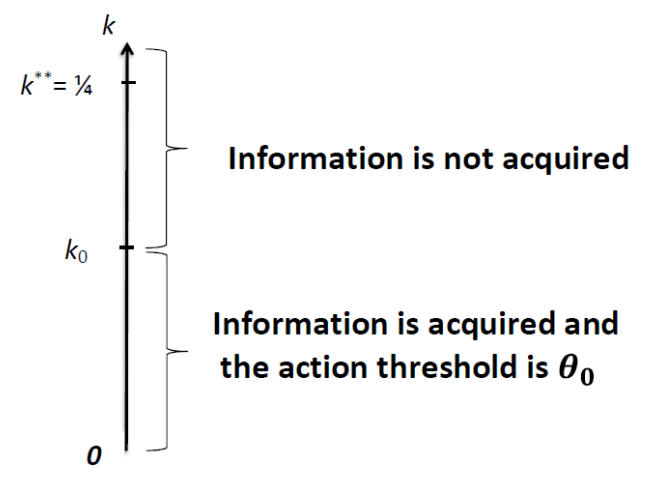

Fig. 2: Privately optimal information acquisition and decision rule

\subsection{Information acquisition with monitoring}

We now introduce a monitor, M. She does not observe whether D acquires information but observes D's action $a$. D's private benefit $b$ and information cost $k$ are common knowledge. After observing $a \mathrm{M}$ may, at a $\operatorname{cost} c>0$, open an investigation, invoking FOI to compel D to reveal his information.

If $\mathrm{D}$ has acquired information then investigation reveals the realisation of $\theta$. If this shows that $\mathrm{D}$ acted contrary to the socially optimal decision rule (1), i.e. the observed $a$ is different from $\alpha^{*}(\theta)$, then a punishment for bias $p \in(0, b)$ is imposed on $\mathrm{D}$ while $\mathrm{M}$ receives a reward $r \geq c$; we denote $c / r \equiv C \leq 1$. Both $p$ and $r$ are assumed to be fixed amounts. ${ }^{4}$ With $p<b$ bias can be deterred only partially; while $p>b$ may be relevant for some

\footnotetext{
${ }^{4}$ The main results also hold in a richer model in which the punishment and reward for bias are proportional to the impact of the biased decision on social welfare.
} 
applications, allowing for this would greatly increase the complexity of the analysis while leaving unchanged the qualitative conclusions that the introduction of FOI worsens information acquisition and that, depending on the value of $k$, lowering $C$ may reduce information acquisition. If investigation instead reveals that $\mathrm{D}$ has not acquired information, then $\mathrm{M}$ remains uninformed about $\theta$; a punishment for negligence $z p$ is then imposed on $\mathrm{D}$ while $\mathrm{M}$ receives a reward $x r$. We assume that $x, z \in(0,1)$ : negligence is considered a lesser offence, and carries a lower reward, than bias.

If informed, D may pre-empt an investigation by voluntarily disclosing $\theta$ at the same time as choosing $a$. We represent this as $\mathrm{D}$ choosing $v(\theta) \in$ $\{0,1\}$ where $v=0$ is no disclosure and $v=1$ is voluntary disclosure. Information is assumed to be 'hard', i.e. $\theta$ cannot be misreported. If disclosure reveals that $\mathrm{D}$ acted contrary to the socially optimal decision rule (1) he still suffers the punishment for bias $p$. We assume that D suffers a small nuisance cost $\varepsilon$ of undergoing an investigation, which may be interpreted as the inconvenience of responding to M's request, even if he is cleared of wrongdoing. ${ }^{5}$ Given $\varepsilon$ an informed D may prefer to reveal his information rather than risk investigation. We assume $\varepsilon$ to be negligible and generally omit it from the calculations, but it appears in proofs and discussions whenever it breaks an indifference tie.

To summarise, the following game is played by $\mathrm{D}$ and $\mathrm{M}$ :

1. Nature chooses the state of the world $\theta$; this is unobservable.

2. D chooses to acquire information with probability $\gamma \in[0,1]$, paying $k$ to learn the realisation of $\theta$; this choice is unobserved by M.

3. D chooses an observable action $a \in\{0,1\}$; if informed, D also chooses

\footnotetext{
${ }^{5}$ While information disclosure might itself be regarded as costly, responding to a FOI request is likely to be more so as this imposes a short deadline for response, under threat of fines, which is likely to increase costs compared with voluntary disclosure (e.g. by taking staff away from normal duties at short notice). Court-based processes are particularly onerous in terms of staff time and may also require external lawyers and advisers to be hired.
} 
whether or not to disclose his information voluntarily, $v \in\{0,1\}$.

4. If information has not been disclosed, $\mathrm{M}$ chooses whether or not to pay $c$ to open an investigation; payoffs are realised.

The equilibrium concept used throughout the paper is perfect Bayesian equilibrium $(\mathrm{PBE})$. We denote by $\underline{\pi}$ the probability that $\mathrm{M}$ investigates after observing action $a=0$ and by $\bar{\pi}$ the probability that she investigates after observing $a=1$. Normalising to zero M's payoff if she does not open an investigation, ${ }^{6}$ her payoff $U^{M}$ from investigating after observing action $a$ is equal to $x r-c$ if $\mathrm{D}$ is uninformed and $r\left|a-\alpha^{*}(\theta)\right|-c$ if $\mathrm{D}$ is informed and the state of the world is $\theta$. We assume $M$ is sufficiently sophisticated that, if information is not disclosed, she can infer from D's equilibrium strategy a posterior on $\theta$, but that in order to generate rewards and punishments she nonetheless needs to investigate to obtain evidence. Therefore, M can use her inference only in deciding whether or not to investigate. ${ }^{7}$

\section{Equilibrium}

The full characterisation of equilibrium in the monitoring game is given in Proposition 5, which is presented in the Appendix together with a formal proof. This section gives an intuitive discussion of the main features of the equilibrium outcomes and analyses the impact of introducing FOI and strengthening the rules to reduce the investigation cost.

\footnotetext{
${ }^{6}$ Notice that, if taken into account by the monitor (e.g. a judge), social welfare from the action that has been taken would appear in the payoff from investigating and from not investigating, thus equating the latter to zero is merely a normalisation.

${ }^{7}$ Good journalistic practice requires evidence to be obtained before allegations are reported in a newspaper, as the publisher may be required to defend the article in court. Court-based mechanisms (such as appeals against regulatory decisions) require evidence to be presented to the judge, who then makes a decision based only on that evidence.
} 


\subsection{Decision-maker's action and disclosure choices}

For any investigation strategy $\{\bar{\pi}, \underline{\pi}\}$ chosen by $\mathrm{M}$, an uninformed $\mathrm{D}$ chooses $a=1$ in equilibrium: the punishment for negligence $z p$ is smaller than $b$, thus $a=1$ is optimal even if this increases the probability of investigation.

When $\mathrm{D}$ is informed, his equilibrium disclosure choice depends on the social optimality of his action and M's investigation strategy. If he takes the socially optimal action, hence will not be punished even if M learns his information, a strictly positive investigation probability causes him voluntarily to disclose his information to avoid the nuisance cost $\varepsilon$. When this probability is zero he is indifferent towards disclosure. When taking the socially inferior action, however, he will not disclose voluntarily unless $\mathrm{M}$ investigates with probability one, in which case the punishment for bias will be suffered anyway and disclosure avoids $\varepsilon$.

When informed, D's equilibrium action choice is $\alpha(\theta)=1$ if $\theta$ is weakly greater than a given threshold and 0 otherwise. While in the benchmark without monitoring the threshold is $\theta_{0}=\frac{1-b}{2}$, in the presence of monitoring it is $\theta_{\bar{\pi}} \equiv \frac{1-b+\bar{\pi} p}{2}$. Intuitively, $\theta_{\bar{\pi}}=\theta_{0}$ for $\bar{\pi}=0$ : if $\mathrm{D}$ expects no investigation after $a=1$ then he follows his privately optimal decision rule. For $\bar{\pi}>0, \theta_{\bar{\pi}}$ is to the right of $\theta_{0}$, closer to the social optimum (one-half). This is the discipline effect: the threat of investigation reduces bias. The discipline effect is strongest when $\bar{\pi}=1$; D's action threshold in this case is denoted $\theta_{1} \equiv \frac{1-b+p}{2} \in\left(\theta_{0}, \frac{1}{2}\right)$. These thresholds are illustrated in Figure 3. Note that, since $p<b$, even when $\bar{\pi}=1$ the decision-maker's private benefit is only partially disciplined and $\theta_{1}$ lies strictly to the left of one-half.

$\begin{array}{llllll}0 & \theta_{0} & \theta_{\bar{\pi}} & \theta_{1} & \frac{1}{2} & 1\end{array}$

Fig. 3: Decision rule with monitoring 
Notice also that the informed D's decision rule in the presence of monitoring is independent of $\underline{\pi}$. At any $\theta$ for which $\alpha(\theta)=0$, it is also true that $\alpha^{*}(\theta)=0$, thus investigation after $a=0$ never results in punishment and $\underline{\pi}>0$ has no effect.

\subsection{Value of information with monitoring}

The value of information acquisition to D depends on M's investigation strategy. M's decision to investigate is taken after observing D's action in the absence of disclosure. For $a=0$ D's optimal decision rule (illustrated in Figure 3) guarantees that this is socially optimal, thus M optimally chooses not investigate: in equilibrium $\underline{\pi}=0$. On observing $a=1 \mathrm{M}$ chooses $\pi$ by comparing the cost of an investigation to its expected reward given her equilibrium belief that either negligence or bias has occurred.

The value of information to $\mathrm{D}$ is found by comparing his payoff from playing $\gamma=0$ and a best response to $\bar{\pi}$ in the subsequent continuation game with his payoff from playing $\gamma=1$ and a best response to $\bar{\pi}$ in the subsequent continuation game. The former value decreases in $\bar{\pi}$ as a higher probability of investigation increases the expected punishment for negligence. The impact of $\bar{\pi}$ on the latter value is more complex: higher $\bar{\pi}$ increases the probability of punishment when taking the socially inferior action, but at the same time the action threshold moves closer to the socially optimal one through the discipline effect, thus the set of types who take the socially inferior action is reduced. As shown in the Appendix, the value of information as a function of $\bar{\pi}$ is the following quadratic:

$$
k_{\bar{\pi}} \equiv \frac{p^{2}}{4} \bar{\pi}^{2}-\frac{p}{2}(b-2 z) \bar{\pi}+k_{0}
$$

It is useful to consider the value of information in the two extreme cases of $\bar{\pi}=0$ and $\bar{\pi}=1$. In Section 2.1 we defined the value of hidden 
information $k_{0}$ as the value of information in the absence of monitoring. This quantity can also be interpreted as the value of information $k_{\bar{\pi}}$ when $\mathrm{M}$ chooses $\bar{\pi}=0$. We now define the value of observed information: this is the maximum cost $\mathrm{D}$ is willing to incur to acquire information when $\bar{\pi}=1$ and is given by

$$
k_{1}=\frac{p^{2}}{4}-\frac{p}{2}(b-2 z)+k_{0}
$$

Looking at the impact of $\bar{\pi}$ on $k_{\bar{\pi}}$ in (2), notice that if $z$ is sufficiently large at $z>\frac{b}{2}, k_{\bar{\pi}}$ is increasing in $\bar{\pi}$ for $\bar{\pi} \in[0,1]$. In this case the negative impact of a higher $\bar{\pi}$ on the payoff from being uninformed dominates the other effects; the minimum value of information is then $k_{0}$ and its maximum is $k_{1}$. If instead $z$ is sufficiently small, with $z<\frac{b-p}{2}, k_{\bar{\pi}}$ decreases in $\bar{\pi}$; then the minimum value of information is $k_{1}$ (which may be negative) and its maximum is $k_{0}$. Finally, for intermediate values $\frac{b-p}{2}<z<\frac{b}{2}$ the value of information is decreasing in $\bar{\pi}$ for small values of $\bar{\pi}$, reaches a (possibly negative) minimum $k_{\min } \equiv k_{0}-\frac{(b-2 z)^{2}}{4}$ at $\bar{\pi}=\frac{b-2 z}{p}$, and increases for larger values of $\bar{\pi}$.

\subsection{Impact of freedom of information}

We now present the equilibria of the game and discuss the impact of FOI. When $k$ exceeds its maximum value $\max \left\{k_{0}, k_{1}\right\}$, identified in Section 3.2, $\mathrm{D}$ does not acquire information for any investigation strategy chosen by M. The discussion therefore focuses on cases where $k$ is no greater than its maximum value.

\subsubsection{Case 1: Small punishment for negligence}

The following proposition (which follows directly from Proposition 5, in the Appendix) describes the case where the punishment for negligence is sufficiently small that the value of information decreases in $\bar{\pi}$, specifically 
$z \leq \frac{b-p}{2} .{ }^{8}$ We define a critical threshold of M's cost-reward ratio, $C_{0} \equiv \frac{b}{1+b}$. The equilibria are shown graphically in Figure 4.

Proposition 2 Equilibrium with small punishment for negligence Assume that $z \leq \frac{b-p}{2}$.

(a) If $k \in\left[\min \left\{0, k_{1}\right\}, k_{0}\right]$ then negligence depends on $C$ as follows:

(i) For $C \leq x$ there exists an equilibrium with $\gamma=0$.

(ii) For $C>x$ there exists a mixed strategy equilibrium with $\gamma \in(0,1)$ and $\gamma$ increasing in $C$. If information is acquired, only types $\theta \geq \frac{1}{2}$ disclose it and the action threshold is $\theta_{\bar{\pi}} \in\left(\theta_{0}, \theta_{1}\right)$, independent of $C$ and decreasing in $k$.

(iii) For $C \geq C_{0}$ there exists an equilibrium with $\gamma=1$, no disclosure, and action threshold $\theta_{0}$.

(b) If $k<k_{1}$ and $k_{1}>0$, then $\gamma=1$ for any $C$. Moreover,

(iv) For any $C$ there exists an equilibrium with full disclosure and action threshold $\theta_{1}$.

(v) For $C \geq C_{0}$ there exists an equilibrium with no disclosure and action threshold $\theta_{0}$.

\footnotetext{
${ }^{8}$ Note that if $p>b$ the RHS of this condition would be negative and this case would not exist.
} 


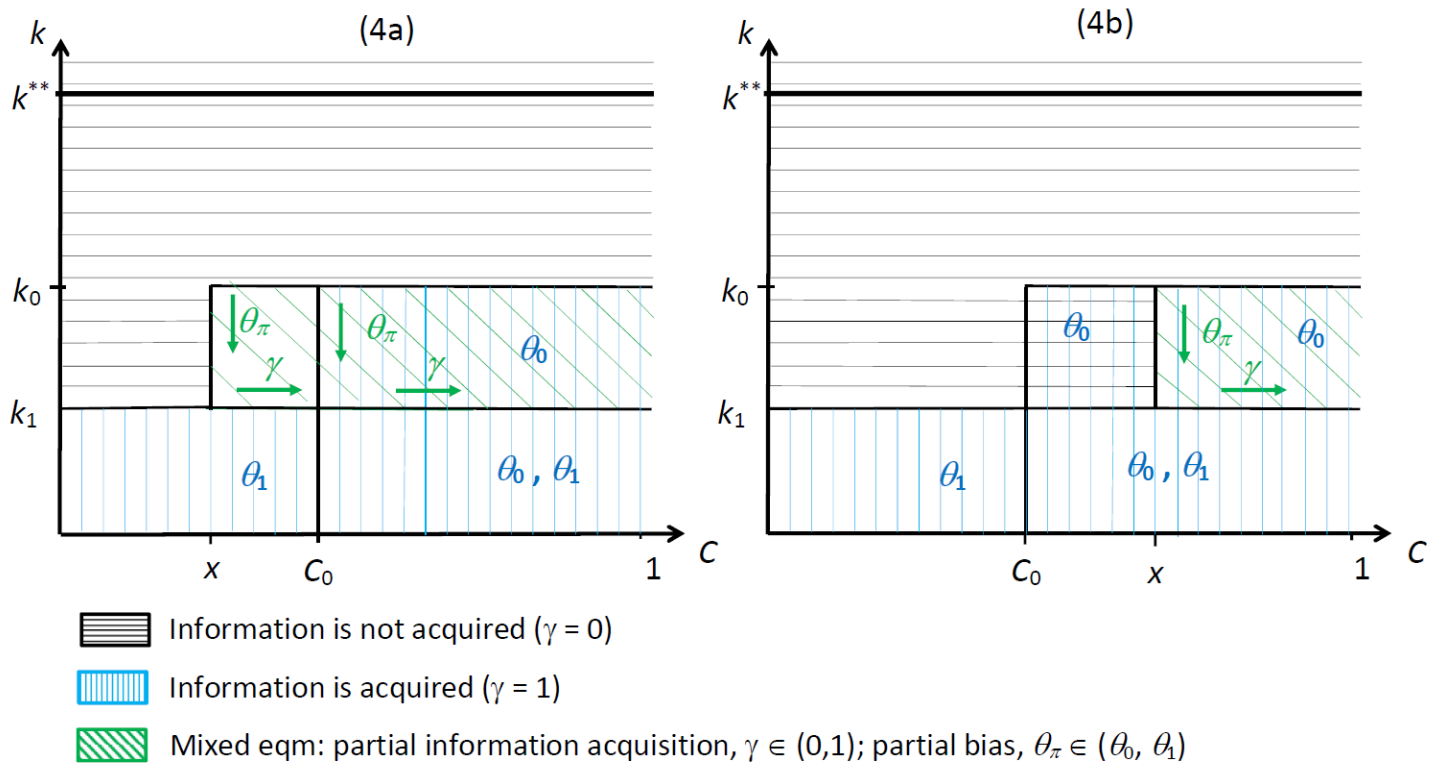

Fig. 4: Equilibria with small punishment for negligence

If the cost of information is in the same range as its value, i.e. $k \in$ $\left[\min \left\{0, k_{1}\right\}, k_{0}\right]$, information acquisition depends on the magnitude of $C$. For $C \leq x$ there exists an equilibrium (i) in which $\mathrm{M}$ investigates after $a=1$ with probability one $(\bar{\pi}=1)$; the value of information is then $k_{1}$, which is lower than its cost and so D does not acquire it.

For $C>x \mathrm{M}$ optimally investigates if and only if she believes with sufficiently high probability that D is informed and biased, as the reward for uncovering negligence is below the cost of investigation. There exists a mixed strategy equilibrium (ii) in which $\gamma \in(0,1)$, increasing in $C$, and $\mathrm{M}$ chooses $\bar{\pi} \in(0,1)$, decreasing in $k$. The intuition is related to well-known results in inspection games. Fix a pair $(C, k)$ for which this equilibrium exists and consider a marginal increase in $C$. To preserve M's indifference the expected reward from investigating after $a=1$ must also increase. In equilibrium, $a=1$ signals either that $\mathrm{D}$ is negligent or that he is informed and biased. Since $x<1$, the second event implies a higher reward for M; thus, when $C$ increases, $\gamma$ must also increase to preserve M's indifference. 
Similarly, consider a marginal increase in $k$. To preserve D's indifference the value of information must also increase. With sufficiently small $z, k_{\bar{\pi}}$ is decreasing in $\bar{\pi}$, hence $\bar{\pi}$ must decrease to preserve indifference.

For $C \geq C_{0}$ a third type of equilibrium (iii) exists in which $\mathrm{D}$ acquires information, does not disclose it and follows the privately optimal threshold $\theta_{0}$. If $\mathrm{M}$ observes $a=1$ she infers from equilibrium beliefs that it might have been taken either by a type in $\left[\theta_{0}, \frac{1}{2}\right)$, in which case investigation would yield a reward, or by a type in $\left[\frac{1}{2}, 1\right]$, in which case there would be

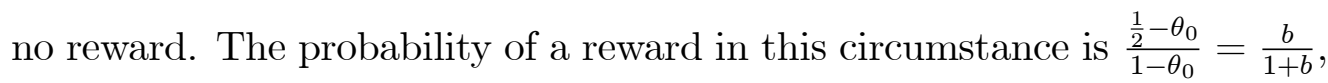
which we denote by $C_{0}$ : if $C$ weakly exceeds this probability $\mathrm{M}$ does not investigate. Since there is no investigation, the value of information is $k_{0}$, which is greater than its cost, hence D optimally acquires information.

If the cost of information is less than its minimum value, $k<k_{1},{ }^{9}$ information acquisition is independent of $C$ and always takes place. For $C \geq C_{0}$ the third equilibrium noted above also exists (cases (iii) and (v) of the proposition refer to the same equilibrium). In addition, for any $C$ there exists an equilibrium (iv) in which D always voluntarily discloses his information when taking $a=1$, thus his action is fully disciplined. To enforce this equilibrium, failure to disclose must prompt an investigation; for any investigation cost there exists at least one set of off-equilibrium beliefs guaranteeing that this is indeed optimal for M. ${ }^{10}$

Impact of FOI. Comparing Figure 4 with the no-monitoring benchmark in Figure 2, with small $z$ the introduction of FOI to permit monitoring increases negligence (the chilling effect) and may reduce bias (the discipline effect). In Figure 2 information is always acquired for $k<k_{0}$, while in Figure 4 it is acquired for sure only for $k<k_{1}<k_{0}$. When infor-

\footnotetext{
${ }^{9}$ Notice that this region does not exist if the parameter values are such that $k_{1}<0$.

${ }^{10}$ The smaller is $C$, the larger the set of off-equilibrium beliefs for which it is optimal for the monitor to investigate.
} 
mation is acquired, in Figure 2 the action threshold is $\theta_{0}$, while in Figure 4 there may be a higher, socially preferable threshold $\left(\theta_{1}\right.$ or $\left.\theta_{\bar{\pi}}\right)$. Bearing in mind that negligence entails that the socially inferior action is taken for all $\theta \in\left[0, \frac{1}{2}\right)$, the quality of decision-making may improve for $k<k_{1}$ but may be worsened for $k \in\left[k_{1}, k_{0}\right]$ as there are now equilibria in which negligence occurs.

The impact of strengthening FOI to reduce $C$ depends on the value of $k$. For $k<k_{1}$ information is always acquired; reducing $C$ below $C_{0}$ may then be beneficial in reducing bias. But for $k \in\left[k_{1}, k_{0}\right]$ strengthening FOI is likely to increase negligence and might also increase bias. Equilibrium (iii), with full information acquisition, exists only for $C>C_{0}$. In the mixed equilibrium (ii) the probability of negligence increases as $C$ is reduced: at $C=x$ the probability of information acquisition falls to zero. For sufficiently low $C$ the only equilibrium is (i), where information is not acquired. Therefore reducing $C$ is likely to deter information acquisition partially or entirely. Meanwhile, reducing $C$ can lower bias only if $x$ is sufficiently low (Figure $4 \mathrm{a}$ ) and $C$ is reduced from a value above $C_{0}$ to a value in $\left(x, C_{0}\right)$. If $x$ is higher (Figure $4 \mathrm{~b}$ ), a reduction in $C$ has either no effect on bias or, if $C$ is reduced from a value above $x$ to a value in $\left(C_{0}, x\right)$, actually increases it. Thus the quality of decision-making is likely to worsen.

\subsubsection{Case 2: Large punishment for negligence}

It might be suggested that information acquisition could be enforced simply by raising $z$ to punish D more severely for negligence. Proposition 3 (which follows directly from Proposition 5) describes equilibrium outcomes when $z$ is large, demonstrating that this approach may not be effective and that, even in this case, lowering $C$ may increase negligence. The proposition considers the case where $z \geq \frac{b}{2}$, so that $k_{\bar{\pi}}$ is increasing in $\bar{\pi}$. Figure 5 
illustrates.

Proposition 3 Equilibrium with large punishment for negligence Assume that $z \geq \frac{b}{2}$.

(a) If $k \in\left[k_{0}, k_{1}\right]$ then negligence depends on $C$ as follows:

(i) For any $C$ there exists an equilibrium with $\gamma=1$, full disclosure, and action threshold $\theta_{1}$.

(ii) For $C>x$ there exists a mixed strategy equilibrium with $\gamma \in(0,1)$ and $\gamma$ increasing in $C$. If information is acquired, only types $\theta \geq \frac{1}{2}$ disclose it and the action threshold is $\theta_{\bar{\pi}} \in\left(\theta_{0}, \theta_{1}\right)$, independent of $C$ and increasing in $k$.

(iii) For $C \geq x$ there exists an equilibrium with $\gamma=0$.

(b) If $k<k_{0}$ then $\gamma=1$ for any $C$. Moreover,

(iv) For any $C$ there exists an equilibrium with full disclosure and action threshold $\theta_{1}$.

(v) For $C \geq C_{0}$, there exists an equilibrium with no disclosure and action threshold $\theta_{0}$.

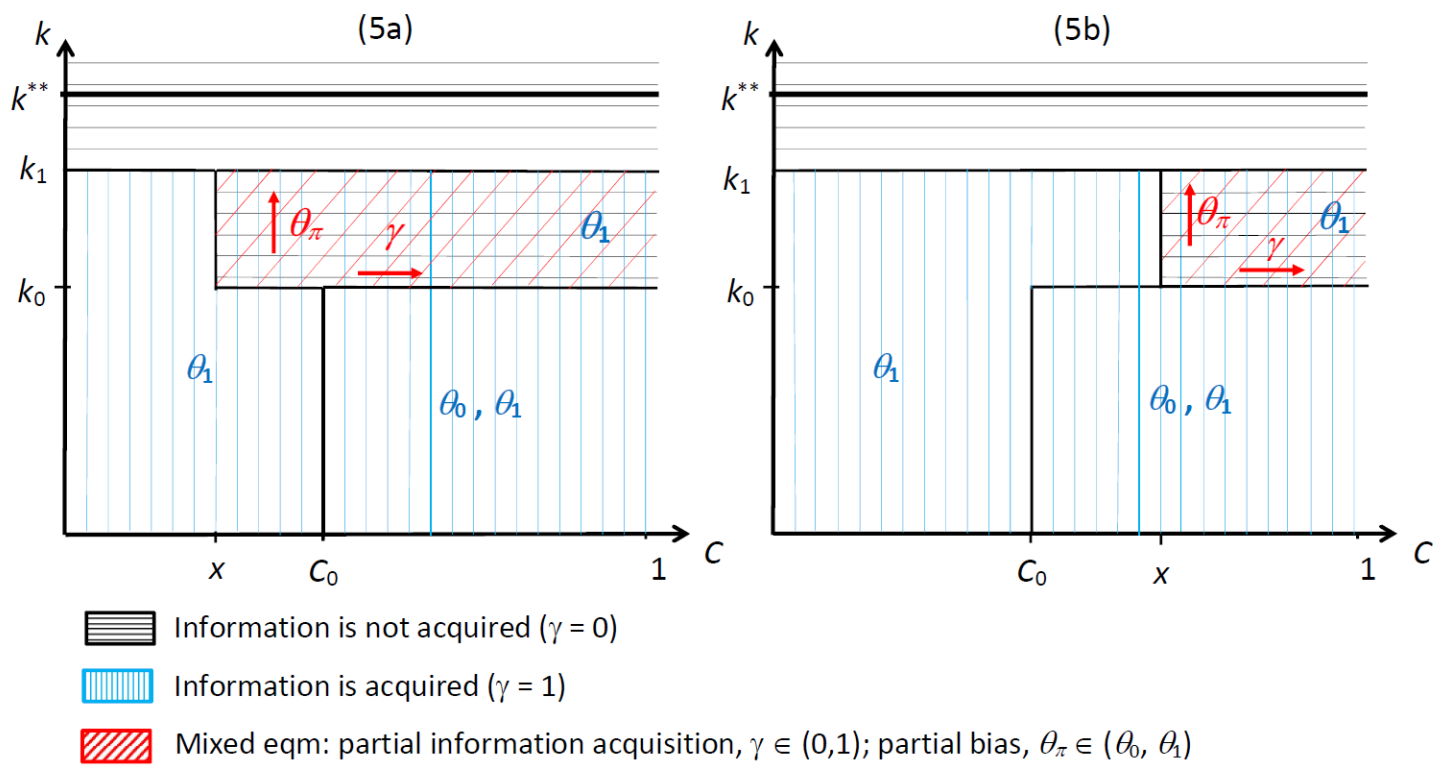

Fig. 5: Equilibria with large punishment for negligence 
For intermediate $k \in\left[k_{0}, k_{1}\right]$ information acquisition again depends on the magnitude of $C$. Cases (i) and (iv) are analogous to case (iv) of Proposition 2 and case (v) is the same as in that proposition. In case (iii), given equilibrium beliefs the cost of investigation exceeds its reward so M does not investigate; the value of information is then $k_{0}$, which is smaller than its cost so D does not acquire information. The mixed equilibrium (ii) is analogous to the mixed equilibrium in Proposition 2 except that now the probability of investigation, and hence the extent of discipline, is increasing rather than decreasing in $k$. As explained above, to preserve D's indifference as $k$ increases, $k_{\bar{\pi}}$ must also increase; since $k_{\bar{\pi}}$ is now increasing in $\bar{\pi}$ this requires $\bar{\pi}$ to increase.

Impact of FOI. Comparing Figures 2 and 5, with large $z$ the introduction of FOI may reduce both negligence and bias. By raising $k_{1}$ above $k_{0}$, large $z$ tends to reduce negligence compared with the no-monitoring benchmark, but the multiplicity of equilibria in the region where $k \in\left[k_{0}, k_{1}\right]$ and $C>x$ means that, even in this case, negligence may still occur.

Strengthening FOI to reduce $C$ has the following effects. As with low $z$, if $k$ is below the minimum value of information (now $k_{0}$ ) negligence never occurs and reducing $C$ may be beneficial in reducing bias. But for $k \in\left[k_{0}, k_{1}\right]$ reducing $C$ may increase negligence and does not necessarily reduce bias. For $C>x$ there are multiple equilibria: if $C$ is reduced but remains greater than $x$, negligence increases in the mixed equilibrium and is unaffected in the other two. Only when $C$ falls below $x$ is negligence eliminated. Meanwhile, a decrease in $C$ reduces bias only if the mixed equilibrium is played initially and $C$ is then reduced below $x$, otherwise bias is unaffected. Bearing in mind that negligence implies an action threshold of zero, if $C$ is reduced but remains above $x$ then the quality of decisionmaking can only worsen, while reducing $C$ below $x$ may improve it. 
To simplify the exposition Propositions 2 and 3 focus on the cases where $z \leq \frac{b-p}{2}$ and $z \geq \frac{b}{2}$ respectively. For completeness we present in Figure 6 the equilibrium outcomes for the intermediate interval $z \in\left(\frac{b-p}{2}, \frac{b}{2}\right)$. The mixed equilibria from Propositions 2 and 3 now co-exist with overlapping existence regions: the former exists for $k \in\left(k_{\min }, k_{0}\right)$ and the latter exists for $k \in\left(k_{\min }, k_{1}\right){ }^{11}$ These coincide when $k_{1}=k_{0}$, which is the case for $z=\frac{2 b-p}{4}$.
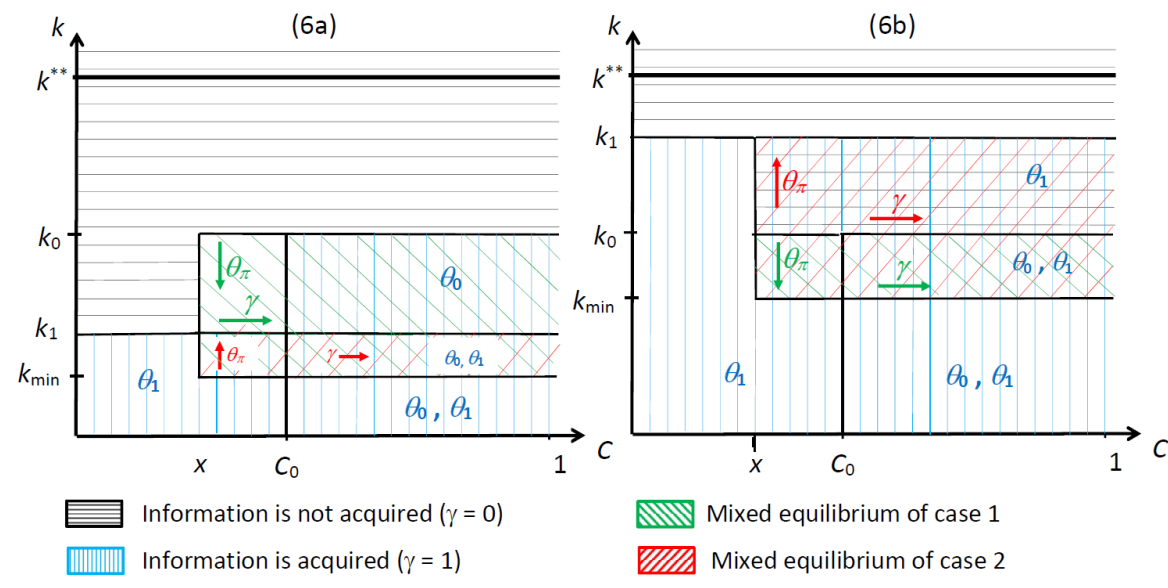

Fig. 6: Equilibria with $z \in\left(\frac{b-p}{2}, \frac{2 b-p}{4}\right)$ (6a) and $z \in\left(\frac{2 b-p}{4}, \frac{b}{2}\right)$ (6b)

\section{Institutional design}

Section 2.1 identified the first best outcome in which information is acquired if and only if its cost does not exceed $k^{* *}$ and the action threshold is onehalf. In this section we ask whether this can be implemented by FOI. The answer depends on which policy instruments the legislator can control. Suppose the legislator can set not only the investigation cost but also all of the punishment and reward parameters in the game between D and M.

\footnotetext{
${ }^{11}$ The indifference condition for $\mathrm{D}$ is a quadratic equation in $\bar{\pi}$. For the values of $z$ considered in Proposition 2 only the smallest solution lies in $[0,1]$. For the values in Proposition 3 only the largest solution is relevant, while for intermediate values both solutions are relevant.
} 
Proposition 4, which follows directly from Proposition 5, gives the values of $(p, z, x, C)$ such that the game has a unique equilibrium which implements the first best outcome. Figure 7 illustrates.

Proposition 4 First best solution

The first best outcome is achieved by setting the parameters as follows: $p^{*}=b ; \quad z^{*}=\frac{1}{2} ; \quad x^{*} \geq C_{0} ; \quad\left(\frac{c}{r}\right)^{*} \equiv C^{*}<C_{0}$

With $p^{*}=b, \theta_{1}$ is equal to the social optimum of one-half. Given that $z^{*}=\frac{1}{2}$ this is a special case of Proposition 3 in which $k_{1}$ is equal to the first best value of information $k^{* *}$. With $C^{*}<C_{0}$ and $x^{*} \geq C_{0}$ the only equilibrium is one in which information is acquired if and only if $k \leq k^{* *}$. When acquired, information is fully disclosed and used according to the socially optimal action rule. ${ }^{12}$ Note that although the conditions on $C$ and $x$ are required for uniqueness, the first best exists for all $C, x \leq 1$.

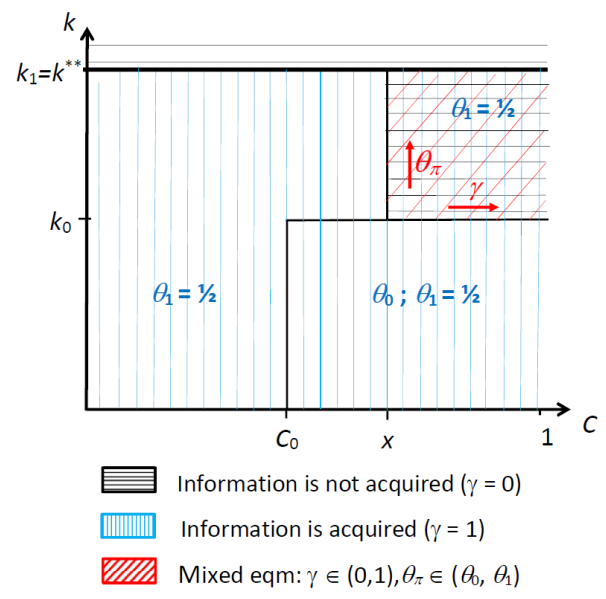

Fig. 7: First best outcome

In practice it is unlikely that the legislator can control all of the reward and punishment parameters. In accordance with the theory of the second best, when it is impossible to set all parameters at their first best levels

\footnotetext{
${ }^{12}$ For $k>k^{* *}$ the observation of $a=1$ without disclosure triggers an investigation even though it is optimal that no information has been acquired. If this is regarded as socially costly this can be avoided by setting $x=0$ when $k>k^{* *}$.
} 
implementing a rule that moves one parameter closer to its first best level might be counterproductive. As Section 3.3 has demonstrated, when the other parameters are not at their first best levels reducing $C$, by either reducing $c$ or increasing $r$, may have unintended consequences in aggravating negligence and/or increasing bias.

Raising $z$, perhaps by introducing an information-gathering requirement as part of the decision-making process with sizeable penalties for failure, ${ }^{13}$ may help in mitigating negligence: higher $z$ increases $k_{\bar{\pi}}$ for any positive $\bar{\pi}$. This raises $k_{1}$ in Figures 4, 5 and 6, and also $k_{\min }$ in Figure 6, in each case expanding the region with full information acquisition at the expense of regions with full or partial negligence.

The desirability of raising $x$, perhaps by introducing payments akin to those paid to whistle-blowers, depends on the magnitude of $z$. When $z$ is small increasing $x$ paradoxically increases negligence by expanding the region with no information acquisition (see Figure 4). Intuitively, a larger reward for uncovering negligence increases the incentive to investigate; if the relative punishment for negligence is small, however, this lowers the value of information. If $z$ is sufficiently large, by contrast, raising $x$ shrinks the region in which there is full or partial negligence (see Figure 5). Accordingly, it is desirable to increase $x$ if and only if $z$ is sufficiently large.

Increasing $p$ may reduce bias: in the equilibrium with minimum bias $\theta_{1}$, larger $p$ moves this threshold closer to the social optimum. However, when $z$ is small an increase in $p$ may reduce $k_{1}$, worsening negligence. This can be seen by differentiating expression (3) with respect to $p$ to obtain $d k_{1} / d p=z-(b-p) / 2$. The first element, which is positive, stems from increasing the absolute value of the punishment for negligence $z p$. The second element, which is negative, is due to the greater discipline imposed

\footnotetext{
${ }^{13}$ For example, decision-making by industry regulators and antitrust authorities in the U.K. has become more formalised than two decades ago, with the collection and publication of considerable amounts of information now required as part of the process.
} 
on the use of information (via $\theta_{1}$ ) and the larger punishment for bias. When the second effect dominates we are in Case 1 (discussed in Section 3.3.1); raising $p$ modestly such that we remain in this case then lowers $k_{1}$, increasing negligence. Otherwise $k_{1}$ increases with $p$ : raising $p$ then becomes unambiguously beneficial, reducing both negligence and bias.

\section{Applications}

We describe three applications of our model, interpreting the players and parameters in each setting and drawing out policy recommendations.

\subsection{Media and political accountability}

News media provide an important check on the behaviour of politicians, holding government to account (for a survey of economics literature on media coverage and political accountability see Strömberg, 2015). Placing our model in this context the players and payoffs can be interpreted as follows. A politician (the decision-maker) takes an action that affects social welfare and may benefit his private interests. A news outlet (the monitor) decides whether or not to investigate. FOI provides easy access to information held by the politician, reducing the cost of researching a story. Penalties and rewards derive from the readership and impact of the story: the news outlet makes a 'scoop' which attracts additional readers, raising profits from reader fees and/or advertising impacts, while the politician is punished by negative publicity. As long as his career is not fatally damaged, the punishment is likely to be less than the private benefit $(p<b)$, news stories tending to pass quickly.

Punishments and rewards are usually higher for bias than for negligence ( $x$ and $z$ are small): bias is a more compelling news story while the difficulty of assessing what information could and should have been obtained makes 
negligence a harder story to convey. With small $z$ the outcome resembles Case 1, illustrated in Figure 4. Since $x$ is also small the existence region for the mixed equilibrium extends far to the left (panel 4a). Accordingly, when $k$ is in the same range as its value the introduction or strengthening of FOI is likely to increase negligence; a large reduction in $C$ may deter information acquisition altogether. Assuming information is obtained, reducing $C$ might reduce bias - a reduction to just below $C_{0}$ may shift the equilibrium from the one with action threshold $\theta_{0}$ to one with partial or full discipline - but this is not guaranteed. This analysis suggests that while news media may be beneficial in reducing bias, the resulting incentives may not generate the ideal monitoring system and in this context strengthening FOI may increase politicians' tendency to avoid acquiring information that might conflict with their preferred actions.

\subsection{Discovery in court proceedings}

Decisions of public bodies may be challenged in court, such as when a regulatory decision is contested by affected companies or other stakeholders. Pre-trial disclosure processes ('discovery') allow the plaintiff to request evidence from the defendant, resulting in disclosure of all relevant information. The players and payoffs in our model can be interpreted as follows. An official (the decision-maker) takes an action that affects social welfare and may give him a private benefit. A plaintiff (the monitor), which may be an affected firm, consumer organisation or lawyers bringing a class action suit, decides whether to appeal the decision to an (unbiased) court. As part of the appeal process, information held by the official is disclosed: more rigorous discovery rules reduce the plaintiff's cost of pursuing an appeal. If the official is found to have taken a biased decision, punishments and rewards occur in the form of fines imposed by the court and damages and costs awarded to the plaintiff (where the latter represent transfers be- 
tween the parties). Assuming that a successful plaintiff fully recovers her costs, $r$ exceeds $c$ and hence $C<1$. The private benefit may well exceed the punishment for bias: e.g. a planning decision may greatly benefit an official who receives a bribe or holds a stake in the land to be developed while the penalty may be relatively small.

With information disclosure, bias may be proven in court. Negligence, on the other hand, is harder to demonstrate. Even if the court determines that the official did not adequately inform himself before taking the decision, the relative punishment and reward for negligence $(z, x)$ are likely to be small: although costs might be awarded, fines and damages are unlikely to be imposed. Thus the situation is likely to resemble Figure 4a of Case 1. Strengthening discovery to reduce $C$ may then undermine information acquisition, worsening the quality of decision-making. This analysis suggests that the threat of court proceedings may need to be combined with higher penalties (e.g. fines) for failure to underpin decisions with adequate information. If such fines raise $z$ sufficiently the outcome will come under Case 2; it could then be beneficial to strengthen discovery and/or increase payments to the plaintiff in case of negligence so that $C<x$; otherwise, such changes may be best avoided.

\subsection{Product safety approval}

Products such as pharmaceuticals require approval by a regulator (the decision-maker) to ensure their safety and effectiveness before they may be marketed or prescribed. The medical community (the monitor) plays an important role in identifying and highlighting harmful side effects. Although acting in the interests of social welfare, the regulator's objective function may be distorted by pressure to grant approval rapidly: for example, Dr Frances Oldham Kelsey, the U.S. Food and Drug Administration (FDA) physician who in 1960 reviewed Richardson-Merrell's application to 
license thalidomide, was denounced by the manufacturer as a fussy, stubborn, unreasonable bureaucrat for demanding further information on the drug's effects. ${ }^{14}$ When medical studies later revealed that thousands of children had suffered severe birth defects from the drug, regulators in several other countries were strongly criticised for failing to subject the drug to sufficient checks, resulting in financial compensation being sought from those countries' governments as well as the drug's manufacturers.

In the context of our model, the private benefit $b$ from granting approval (arising from either positive inducements or the absence of a negative payoff, $-b$, representing criticism such as that applied to Dr Kelsey) may exceed the punishments for bias and negligence. In this example, in contrast to the previous two, the penalty for negligence may be fairly large, bringing $z$ close to 1 . Rewards to medical professionals, whose motivation is to protect their patients, will be similar for negligence and bias, i.e. $x$ is also close to 1 . With high $z$ and $x$ the equilibrium will be as represented in Figure $5 \mathrm{~b}$ of Case 2. For intermediate values of $k$, if $C$ is initially high and the mixed equilibrium is played a small reduction in $C$ within the mixed strategy region worsens information acquisition, but a larger reduction which shifts the equilibrium to one in which full information is acquired is beneficial.

\section{Conclusion}

The paper has considered the impact of freedom of information (FOI) rules on the quality of decision-making in a setting where a decision-maker with a private benefit from one action may acquire information prior to taking the decision. We identify a 'chilling effect' of FOI on information acquisition by the decision-maker (or, equivalently, on effort put into analysing

\footnotetext{
${ }^{14}$ Dr Kelsey's persistence largely spared the U.S. from the thalidomide tragedy. See McFadden (2015) and Bernstein and Sullivan (2015).
} 
information), increasing negligence, and a 'discipline effect' on the use of information, reducing the extent of bias in favour of the preferred action. We find that strengthening FOI may increase negligence and, in some cases, even increases bias. While increasing the punishment for negligence may reduce its prevalence, raising the monitor's reward for discovering it may paradoxically increase negligence, if the associated punishment is too small. Finally, we have interpreted our model in the context of the media and political accountability, court proceedings and product safety approval, drawing out lessons for institutional design.

Our model has a number of testable implications. With suitable measures of information acquisition, one could test directly the prediction that the introduction or strengthening of FOI may reduce information acquisition prior to policy decisions: such measures might include the time taken to consider the matter or the manpower devoted to the project (assuming such data can be obtained from public bodies with the appropriate degree of granularity). An alternative approach would be to test the variance of policy outcomes and the accuracy of published preditions following the introduction or strengthening of FOI: if information is not acquired, the socially inferior action is taken in states where its negative impact on social welfare is greater, thus the variability of policy outcomes will increase. In addition, published predictions will be less informed, implying that their accuracy is likely to decline.

While disclosure is not the main focus of discussion in the paper, the model's implications in this area might also be tested. In some parameter regions strengthening FOI increases the likelihood that an informed decision-maker discloses information voluntarily. Since, by definition, information disclosure is publicly observable, its occurrence and extent should be relatively easy to measure. If information disclosure were found to increase following the introduction or strengthening of FOI (without contem- 
poraneous strengthening of publication requirements) this would provide evidence in support of the mechanisms highlighted in the paper.

\section{Appendix: Full equilibrium characterisation}

We start by calculating the value of information $k_{\bar{\pi}}$ defined in Section 3.2:

$$
\begin{aligned}
k_{\bar{\pi}} & =\int_{0}^{\theta_{\bar{\pi}}}-\theta d \theta+\int_{\theta_{\bar{\pi}}}^{1}(b-(1-\theta)) d \theta-\int_{\theta_{\bar{\pi}}}^{1 / 2} \bar{\pi} p d \theta-\left(\int_{0}^{1}[b-(1-\theta)] d \theta-\bar{\pi} z p\right) \\
& =\frac{p^{2}}{4} \bar{\pi}^{2}-p\left(\frac{b-2 z}{2}\right) \bar{\pi}+k_{0}
\end{aligned}
$$

Next, denote by $s_{1}$ the measure of the set of types choosing $a=1$ and $v=0$ in equilibrium, and by $\underline{s_{1}}$ the measure of the subset of $s_{1}$ comprising types $\theta<\frac{1}{2}$.

Proposition 5 Full equilibrium characterisation

- An equilibrium in which $\gamma=0$ exists for $k \geq k_{\bar{\pi}}$, with $\bar{\pi}=1$ if $C<x$, $\bar{\pi} \in[0,1]$ if $C=x$, and $\bar{\pi}=0$ if $C>x$. D chooses $a=1$.

- An equilibrium in which $\gamma=1, \bar{\pi}=1$, and D chooses $v=1$ whenever choosing $a=1$ exists for $k \leq k_{1}$. The action threshold is $\theta_{1}$.

- An equilibrium in which $\gamma=1, \bar{\pi}=0$, and some or no types choose $v=1$ when choosing $a=1$ exists for $k \leq k_{0}, C \geq \frac{s_{1}}{s_{1}} \in\left[C_{0}, 1\right]$. The action threshold is $\theta_{0}$.

- An equilibrium in which $\gamma \in(0,1), \bar{\pi}=1$, and an informed $D$ chooses $v=1$ whenever choosing $a=1$ exists for $k=k_{1}$ and $C \leq x$. The action threshold is $\theta_{1}$. If uninformed, $D$ chooses $a=1$.

- An equilibrium in which $\gamma \in(0,1), \bar{\pi}=0$, and some or no types of informed $D$ choose $v=1$ when choosing $a=1$ exists for $k=k_{0}$. The following condition holds: $C \geq \frac{\gamma s_{1}+(1-\gamma) x}{\gamma s_{1}+(1-\gamma)}$. The action threshold is $\theta_{0}$. If uninformed, $D$ chooses $a=1$. 
- Equilibria in which $\gamma \in(0,1), \bar{\pi} \in(0,1)$, informed types with $\theta \geq \frac{1}{2}$ choose $v=1$ and the remaining types choosing $a=1$ choose $v=0$ are such that $\gamma=\frac{2(C-x)}{2(C-x)+(b-\bar{\pi} p)(1-C)}$. This equals zero for $C=x$, is increasing in $C$ and equal to one for $C=1$. For $z<\frac{b-p}{2}$, an equilibrium in this class exists for $k \in\left(k_{1}, k_{0}\right)$ and $\bar{\pi}$ is decreasing in $k$. For $\frac{b-p}{2} \leq z \leq \frac{b}{2}$, there exists an equilibrium with $\bar{\pi}$ increasing in $k$ for $k \in\left(\max \left\{0, k_{\min }\right\}, k_{1}\right)$ and one with $\bar{\pi}$ decreasing in $k$ for $k \in\left(\max \left\{0, k_{\min }\right\}, k_{0}\right)$. For $z>\frac{b}{2}$, an equilibrium in this class exists for $k \in\left(k_{0}, k_{1}\right)$ and $\bar{\pi}$ is increasing in $k$. The action threshold in each case is $\theta_{\bar{\pi}}$. If uninformed, $D$ chooses $a=1$.

- In all the equilibria with $\gamma=1$ or $\gamma \in(0,1)$, either some or no types who choose $a=0$ choose $v=1$, in which case $\underline{\pi}=0$, or all the types who choose $a=0$ choose $v=1$, in which case $\underline{\pi}$ can take any value in $[0,1]$. In the equilibrium with $\gamma=0, \underline{\pi}$ can take any value in $[0,1]$.

\section{Proof.}

Equilibria with $\gamma=0$. In equilibrium, D chooses $a=1$. Given M's equilibrium beliefs, $\bar{\pi}$ depends on the comparison of the expected cost $c$ and the expected reward $x r$. If $C>x$, then $\bar{\pi}=0$. If $C<x$, then $\bar{\pi}=1$. If $C=x$, then $\bar{\pi} \in[0,1]$. A deviation to $\gamma=1$ is undetectable by $\mathrm{M}$ unless $\mathrm{D}$ chooses $a=0$. The value of $\underline{\pi}$ depends on M's off-equilibrium path beliefs following $a=0$, and on the value of $C$. Nonetheless, for any $\underline{\pi}$ D's optimal action threshold after the deviation is $\theta_{\bar{\pi}}$ and the highest payoff gain achievable by deviating to $\gamma=1$ and using the optimal disclosure rule described in Section 3.1 is $k_{\bar{\pi}}-k$. Hence, we can conclude that equilibria with $\gamma=0$ exist iff $k \geq k_{\bar{\pi}}$.

Equilibria with $\gamma=1$. Suppose that $\bar{\pi}=1$. All types taking $a=1$ choose $v=1$ to avoid the nuisance cost and $\mathrm{D}$ follows action threshold $\theta_{1}$. M's off-equilibrium path beliefs after observing $a=1, v=0$ must be such that investigation is optimal for any cost. Notice that $\bar{\pi}=1$ minimises the 
payoff from a deviation to $\gamma=0$, which is unprofitable if $k \leq k_{1}$.

Next suppose that $\bar{\pi}=0$. Types smaller than $\frac{1}{2}$ choosing $a=1$ do not disclose while types above $\frac{1}{2}$ choosing $a=1$ are indifferent towards disclosure. Therefore $\mathrm{D}$ follows action threshold $\theta_{0}$. Since the value of information for $\bar{\pi}=0$ is $k_{0}$, deviation to $\gamma=0$ is unprofitable iff $k \leq k_{0}$. For $\mathrm{M}, \bar{\pi}=0$ is compatible with equilibrium iff $C \geq \frac{s_{1}}{s_{1}}$, the equilibrium probability that investigation after $a=1$ results in a reward. As the proportion of types $\theta \geq \frac{1}{2}$ that do not disclose converges to $1, \frac{s_{1}}{s_{1}}$ converges to $C_{0} \equiv \frac{b}{1+b}$; as the same proportion converges to $0, \frac{s_{1}}{s_{1}}$ converges to 1 .

Finally, to prove that there are no equilibria with $\gamma=1$ and $\bar{\pi} \in(0,1)$, notice that in such an equilibrium all types $\theta \geq \frac{1}{2}$ choosing $a=1$ would disclose, while all types $\theta<\frac{1}{2}$ choosing $a=1$ would not disclose. The action threshold would be $\theta_{\bar{\pi}}$. Given equilibrium beliefs, $M$ would then have an incentive to deviate to $\bar{\pi}=1$.

Equilibria with $\gamma \in(0,1)$. Suppose that $\bar{\pi}=1$. All informed types choosing $a=1$ disclose $\theta$ to save $\varepsilon$ and $\mathrm{D}$ follows action threshold $\theta_{1}$. Under equilibrium beliefs $\mathrm{M}$ attributes $a=1, v=0$ to an uninformed decisionmaker, hence $\bar{\pi}=1$ is optimal for her iff $C \leq x$. The value of information is $k_{1}$, hence $\mathrm{D}$ is indifferent between $\gamma=0$ and $\gamma=1$ iff $k=k_{1}$.

Next suppose that $\bar{\pi}=0$. Types smaller than $\frac{1}{2}$ choosing $a=1$ do not disclose, while types above $\frac{1}{2}$ choosing $a=1$ are indifferent between disclosing or not. Therefore, D follows action threshold $\theta_{0}$. Since the value of information for $\bar{\pi}=0$ is $k_{0}$, D is indifferent between $\gamma=0$ and $\gamma=1$ iff $k=k_{0}$. Given M's equilibrium beliefs, the condition for $\bar{\pi}=0$ to be optimal is that $C \geq \frac{\gamma s_{1}+(1-\gamma) x}{\gamma s_{1}+(1-\gamma)}$, where the exact values of $s_{1}$ and $\underline{s_{1}}$ depend on the disclosure choice of the types $\theta \in\left[\frac{1}{2}, 1\right]$. If none of them discloses, $\frac{\gamma s_{1}+(1-\gamma) x}{\gamma s_{1}+(1-\gamma)}=\frac{\gamma \frac{b}{2}+(1-\gamma) x}{\gamma \frac{1+b}{2}+(1-\gamma)}$, while if they all disclose it is equal to $\frac{\gamma s_{1}+(1-\gamma) x}{\gamma s_{1}+(1-\gamma)}=\frac{\gamma \frac{b}{2}+(1-\gamma) x}{\gamma \frac{b}{2}+(1-\gamma)}$. Notice that $\frac{\gamma s_{1}+(1-\gamma) x}{\gamma s_{1}+(1-\gamma)}=x$ for $\gamma=0$ and $\frac{\gamma s_{1}+(1-\gamma) x}{\gamma s_{1}+(1-\gamma)}=\frac{s_{1}}{s_{1}}$ for $\gamma=1$. 
Finally, suppose that $\bar{\pi} \in(0,1)$. Types smaller than $\frac{1}{2}$ choosing $a=1$ do not disclose, while types above $\frac{1}{2}$ choosing $a=1$ disclose. Therefore $\mathrm{D}$ follows action threshold $\theta_{\bar{\pi}}$. After observing $a=1, v=0$, M, who holds equilibrium beliefs, compares the investigation cost $c$ to the expected reward $\frac{\gamma\left(\frac{1}{2}-\theta_{\bar{\pi}}\right) r+(1-\gamma) x r}{\gamma\left(\frac{1}{2}-\theta_{\bar{\pi}}\right)+(1-\gamma)}$. Therefore $\mathrm{M}$ is indifferent between investigating and not investigating iff $C=\frac{\gamma \frac{b-\bar{\pi} p}{2}+(1-\gamma) x}{\gamma \frac{b-\bar{\pi} p}{2}+(1-\gamma)}$, i.e. iff $\gamma=\frac{2(C-x)}{2(C-x)+(b-\bar{\pi} p)(1-C)}$. This probability is equal to zero for $C=x$. Given $x<1, \gamma$ is increasing in $C$ and equal to one for $C=1$.

The investigation probability $\bar{\pi}$ is determined by the indifference condition for $\mathrm{D}$ at the information acquisition stage: $k=k_{\bar{\pi}}$, which gives the following quadratic equation in $\bar{\pi}$ :

$$
\frac{p^{2}}{4} \bar{\pi}^{2}-\frac{p}{2}(b-2 z) \bar{\pi}+k_{0}-k=0 .
$$

The two solutions to $(4)$ are $\frac{(b-2 z) \pm \sqrt{(b-2 z)^{2}+4\left(k-k_{0}\right)}}{p}$. Notice that real roots exist only for $k \geq k_{\min } \equiv k_{0}-\frac{(b-2 z)^{2}}{4}$ : the cost of information can be equal to its value only if it is larger than the minimum value. Let $\bar{\pi}_{H}(k)$ denote the larger root and $\bar{\pi}_{L}(k)$ denote the smaller root. There are three cases to consider.

Case (a): $z \leq \frac{b-p}{2}$. In this region $k_{\bar{\pi}}$ is strictly decreasing in $\bar{\pi}$ for $\bar{\pi} \in[0,1]$ - We can eliminate the larger root of (4) because it is larger than one. Therefore the equilibrium probability of investigation is $\bar{\pi}_{L}(k)$, which is decreasing in $k$, equal to zero at $k_{0}$ and unity at $k_{1}$.

Case (b): $z \in\left(\frac{b-p}{2}, \frac{b}{2}\right)$. In this region, $k_{\min } \leq \min \left\{k_{0}, k_{1}\right\}$ and $k_{1}>k_{0}$ iff $z>\frac{2 b-p}{4} . \bar{\pi}_{L}(k)$ is decreasing in $k$; it takes value zero at $k=k_{0}$ and its maximum value is $\frac{b-2 z}{p}$ for $k=k_{\min } \cdot \bar{\pi}_{H}(k)$ is instead increasing in $k$; its minimum value is $\frac{b-2 z}{p}$ for $k=k_{\min }$ and it achieves value one for $k=k_{1}$. Within the interval $z \in\left(\frac{b-p}{2}, \frac{b}{2}\right)$, there are two mixed strategy equilibria. The first one exists only for $k \in\left(\max \left\{0, k_{\min }\right\}, k_{0}\right)$ and has probability of 
investigation $\bar{\pi}_{L}(k)$. The second one exists only for $k \in\left(\max \left\{0, k_{\min }\right\}, k_{1}\right)$ and has probability of investigation $\bar{\pi}_{H}(k)$.

Case (c): $z \geq \frac{b}{2}$. In this region $k_{\bar{\pi}}$ is strictly increasing in $\bar{\pi}$ for $\bar{\pi} \in[0,1]$ .The smaller root $\bar{\pi}_{L}(k)$ is strictly negative. Hence, the equilibrium probability of investigation after $a=1, v=0$ is $\bar{\pi}_{H}(k)$, which is increasing in $k$, equal to zero at $k_{0}$ and unity at $k_{1}$.

Value of $\underline{\pi}$. The only equilibria where $a=v=0$ is observed by $\mathrm{M}$ on equilibrium path are those with $\gamma>0$ and partial disclosure by informed types smaller than $\theta_{\bar{\pi}}$. These types are taking the socially optimal action, hence the best response is $\underline{\pi}=0$. In turn, $\underline{\pi}=0$ makes these types indifferent between disclosing and not disclosing. In equilibria where $a=v=0$ is observed only off equilibrium path, any value of $\underline{\pi}$ can be sustained by appropriate off-equilibrium path beliefs.

\section{References}

[1] Allen, Kate, and Jim Pickard (2016), "Ministers retreat from move to weaken freedom of information law," Financial Times, 27 January 2016 .

[2] Bernstein, Adam and Patricia Sullivan (2015), "Frances Oldham Kelsey, FDA scientist who kept thalidomide off U.S. market, dies at 101," The Washington Post, 7 August 2015.

[3] Cabinet Office (2016), "Independent Commission on Freedom of Information Report," March 2016.

[4] Che, Yeon-Koo, and Navin Kartik (2009), "Opinions as incentives," Journal of Political Economy, 117(5), 815-860.

[5] Felgenhauer, Mike, and Petra Loerke (2017), "Bayesian persuasion with private experimentation," International Economic Review, 58(3), 829-856.

[6] Felgenhauer, Mike, and Elisabeth Schulte (2014), "Strategic private experimentation," American Economic Journal: Microeconomics, 6(4), 74-105. 
[7] Frankel, Maurice (2001), "Freedom of information: Some international characteristics," Campaign for Freedom of Information.

[8] Hagenbach, Jeanne, Frederic Koessler and Eduardo Perez-Richet (2014), "Certifiable pre-play communication: Full disclosure," Econometrica, 82(3), 1093-1131.

[9] Hansen, Stephen, Michael McMahon and Andrea Prat (2017), "Transparency and deliberation within the FOMC: A computational lingustics approach," Quarterly Journal of Economics, 133(2), 801-870.

[10] Hazell, Robert, Gabrielle Bourke and Benjamin Worthy (2012), "Open house? Freedom of information and its impact on the UK parliament," Public Administration, 90(4), 901-921.

[11] Hazell, Robert, and Mark Glover (2011), "The impact of freedom of information on Whitehall," Public Administration, 89(4), 1664-1681.

[12] Holmström, Bengt (1979), "Managerial incentive problems: A dynamic perspective," Review of Economic Studies, 6(1), 169-182.

[13] Hoppe, Eve (2013), "Observability of information acquisition in agency models," Economic Letters, 19, 104-107.

[14] Kamenica, Emir, and Matthew Gentzkow (2011), "Bayesian persuasion", American Economic Review, 101(6), 2590-2615.

[15] Kamenica, Emir, and Matthew Gentzkow (2012), "Disclosure of endogenous information," Economic Theory Bulletin, forthcoming.

[16] Levy, Gilat (2007), "Decision making in committees: Transparency, reputation, and voting rules," American Economic Review, 97(1), 150168.

[17] Maskin, Eric, and Jean Tirole (2004), "The politician and the judge: Accountability in government," American Economic Review, 94(4), 1034-1054.

[18] Mathis, Jerome (2008), "Full revelation of information in SenderReceiver games of persuasion," Journal of Economic Theory, 143, 57184 .

[19] McFadden, Robert D.(2015), "Frances Oldham Kelsey, who saved U.S. babies from thalidomide, dies at 101," The New York Times, 7 August 2015. 
[20] Meade, Ellen E., and David Stasavage (2008), "Publicity of debate and the incentive to dissent: Evidence from the US Federal Reserve," The Economic Journal, 118 (April), 695-717.

[21] McIntosh, Toby (2014), "Paraguay is 100th nation to pass FOI law, but struggle for openness goes on," The Guardian, 19 September 2014.

[22] Milgrom, Paul (1981), "Good news and bad news: Representation theorem and applications," Bell Journal of Economics, 12, 380-91.

[23] Milgrom, Paul (2008), "What the seller won't tell you: Persuasion and disclosure in markets", Journal of Economic Perspectives, 22(2), 115-131.

[24] Milgrom, Paul, and John Roberts (1986), "Relying on the information of interested parties", Rand Journal of Economics, 17, 18-32.

[25] Pickard, Jim, and Kiran Stacey (2015), "Freedom of Information is Mission Impossible for Downing St emails," Financial Times, 16 June 2015 .

[26] Prat, Andrea (2005), "The wrong kind of transparency," American Economic Review, 95(3), 862-877.

[27] Quinn, Ben (2016), "Review decides not to change Freedom of Information Act," The Guardian, 1 March 2016.

[28] Rayo, Luis, and Ilya Segal (2010), "Optimal information disclosure," Journal of Political Economy, 118(5), 949-987.

[29] Strömberg, David (2015), "Media coverage and political accountability: Theory and evidence," Handbook of Media Economics, Volume 1A, edited by Simon Anderson, David Strömberg and Joel Waldfogel, Elsevier North-Holland.

[30] The Washington Post (2016), "FOIA at 50," 3 July 2016.

[31] Worthy, Benjamin, and Robert Hazell (2013), "The impact of the Freedom of Information Act in the UK," in Nigel Bowles, James T Hamilton and David Levy (eds.), Transparency in Politics and the Media: Accountability and Open Government, London: L.B. Tauris, $31-45$. 